Research Paper

\title{
AKT-STAT3 Pathway as a Downstream Target of EGFR Signaling to Regulate PD-L1 Expression on NSCLC cells
}

\author{
Sherif Abdelhamed, Keisuke Ogura, Satoru Yokoyama, Ikuo Saiki and Yoshihiro Hayakawa ${ }^{\bowtie}$ \\ Division of Pathogenic Biochemistry, Institute of Natural Medicine, University of Toyama, Sugitani, Toyama, Japan. \\ $\triangle$ Corresponding author: Yoshihiro Hayakawa, Associate Professor, Division of Pathogenic Biochemistry, Department of Bioscience, Institute of Natural \\ Medicine, University of Toyama, Sugitani 2630, Toyama, Toyama, Japan 930-0194. Tel: +81-76-434-7636; Fax: +81-76-434-5058; Email: haya@inm.u-toyama.ac.jp \\ (C) Ivyspring International Publisher. Reproduction is permitted for personal, noncommercial use, provided that the article is in whole, unmodified, and properly cited. See \\ http://ivyspring.com/terms for terms and conditions.
}

Received: 2015.12.16; Accepted: 2016.06.10; Published: 2016.07.18

\begin{abstract}
While cancer development and progression can be controlled by cytotoxic $T$ cells, it is also known that tumor-specific CD8+T cells become functionally impaired by acquiring a group of inhibitory receptors known as immune checkpoints. Amongst those, programmed death-1 (PD-1) is one of the most recognized negative regulators of $T$ cell function. In non-small lung cancers (NSCLCs), the aberrant activation of epidermal growth factor receptor (EGFR) is known to induce PD-LI expression and further the treatment with gefitinib, a tyrosine kinase inhibitor (TKI) for EGFR, decrease the expression of PD-LI on NSCLC. Given the acquired resistance to gefitinib treatment frequently observed by developing secondary-site mutations limiting its efficacy, it is important to understand the downstream mechanism of activated-EGFR signaling for regulating PD-LI in NSCLC. In this study, we demonstrated that AKT-STAT3 pathway could be a potential target for regulating the surface expression of PD-LI on NSCLCs with aberrant EGFR activity and, further, the inhibition of AKT or STAT3 activity could down-regulate the expression of PD-LI even in gefitinib-resistant NSCLCs. These results highlight an importance of AKT-STAT3 pathway as a promising target for potentiating anti-tumor immune responses by regulating PD-LI expression on cancer cells with aberrant EGFR activity.
\end{abstract}

Key words: non-small lung cancer, EGFR, AKT, STAT3, PD-L1

\section{Introduction}

It has been widely appreciated that the immune system can recognize and control nascent transformed cancer cells known as the process called cancer immune-surveillance $[1,2]$. While cytotoxic $\mathrm{T}$ cells recognize tumor-specific antigens and consequently eliminate cancer cells, it is also known that the antigen-specific $\mathrm{CD}^{+} \mathrm{T}$ cells become functionally exhausted upon chronic stimulation [3]. Among the mechanism of such $\mathrm{T}$ cell exhaustion, a group of inhibitory receptors known as immune checkpoints that negatively regulate $\mathrm{T}$ cell function has been extensively explored [4]. The molecule called programmed death-1 (PD-1) is one of the most recognized negative regulators of $T$ cell function [5]. In the context of cancer, specific genomic subsets and oncogenic mutations are known to correlate to the expression level of PD-L1 on cancer cells, which is a specific ligand for PD-1, leading to T cells inactivation and subsequent escape from immune-surveillance [6-8]. Given the importance of the interaction between PD-1 and its ligands for cancer cells escaping from the host immune responses, an inhibition of PD-1/PD-L1 pathway has been shown to regain the effector function of antigen-specific T cells against cancer cells $[5,9]$.

Non-small cell lung cancers (NSCLCs) are the most common and aggressive type of lung cancer with a high mortality rate $[10,11]$. In concert with their high malignancy, NSCLCs are known to express high levels of PD-L1 that contribute to their poor prognosis and immune suppression status $[12,13]$. In addition to its success in melanoma treatment $[14,15]$, clinical studies of PD-1 or PD-L1 targeted therapy by using monoclonal antibodies in patients with advanced or metastatic NSCLCs have been successfully conducted [4,16, 17]. Considering such 
responses of NSCLCs to PD-1 checkpoint therapy, the manipulation of PD-1/PD-L1 interaction can be an attractive strategy to overcome cancer immune escape in NSCLCs.

While a group of oncogenic mutations have been known to correlate to PD-L1 expression on cancer cells $[6,7,18,19]$, the aberrant activation of epidermal growth factor receptor (EGFR) signaling in NSCLCs driven by a mutation of EGFR can be responsible for evading host immune responses through up-regulation of PD-L1 expression on NSCLCs $[8,20]$. EGFR is a member of the ErbB family of receptor tyrosine kinases and its mutations have been regarded as a hallmark of NSCLC progression [21]. EGFR mutations cause the constitutive activation of its downstream signaling pathways associated with cellular proliferation and survival, which include mitogen-activated protein kinases (MAPKs), phosphoinositide 3-kinase (PI3K) and signal transducer and activator of transcription 3 (STAT3) pathways [22, 23]. The small molecule inhibitors for EGFR-tyrosine kinase, such as gefitinib, have been developed for treating cancer cells bearing exon 19 deletions or L858R point mutation of EGFR that render sensitivity to those tyrosine kinase inhibitors (TKIs) [23]. Despite an initial response to gefitinib, the disease often relapses because NSCLCs show a resistance against gefitinib treatment by acquiring a secondary T790M mutation in EGFR [24-26], therefore it is important to explore an alternative strategy for treating those drug-resistant cancer cells.

In this study we sought to determine the important downstream pathway in EGFR signaling which regulates the expression of PD-L1 on NSCLCs for developing a new approach to enhance anti-tumor immune responses by modulating a PD- 1 immune checkpoint. Our present results indicate that AKT-STAT3 pathway can be a potential target for regulating a surface expression of PD-L1 on NSCLCs with aberrant EGFR activity. We further demonstrated the inhibition of either AKT or STAT3 could down-regulate the expression of PD-L1 even in gefitinib-resistant NSCLCs. These results highlight an importance of AKT-STAT3 pathway as a promising target for potentiating anti-tumor immune responses by regulating PD-L1 expression on cancer cells with an aberrant EGFR activity.

\section{Materials and Methods}

\section{Reagents}

The EGFR-TKI (Gefitinib) was purchased from Cayman Chemical (MI, USA). The AKT inhibitor (MK-2206) was purchased from Active Biochemicals (Wan Chai, Hong Kong). The PI3K inhibitor
(LY-294002), the MEK inhibitor (U0126), the JNK-inhibitor (SP600125) and the EGFR-TKI (PD153035) were purchased from Merck Millipore (MA, USA). The JSI-124 was purchased from Sigma Aldrich (MO, USA). The mTOR inhibitor (Rapamycin) was purchased from Santa Cruz Biotechnologies (CA, USA). Recombinant human EGF was obtained from R\&D System, and HGF was purchased from Pepro Tech (NJ, USA). All of the chemical inhibitors were dissolved in DMSO and the final concentration of DMSO was less than $0.1 \%$. Primary antibodies against pEGFR (Y1068), pAKT (Ser473), STAT3, pSTAT3 (tyr705), pSTAT3 (ser727), and pERK (Thr-202, Tyr-204) were obtained from Cell Signaling Technology (MA, USA). Antibodies against EGFR, PCNA, AKT, $\alpha$-Tubulin and $\beta$-actin were obtained from Santa Cruz Biotechnologies (CA, USA).

\section{Cell lines}

PC-9 and R-PC-9 cells were kind gifts from Dr. Kiura (Okayama University, Japan). LUt-99, A549 and PC-14 cells were obtained from the American Type Culture Collection (ATCC), MD, USA. All cells were cultured in RPMI-1640 medium (Invitrogen, CA, USA). Media were supplemented with $10 \%$ heat-inactivated fetal calf serum, $2 \mathrm{mM}$ L-glutamine, 100 units $/ \mathrm{ml}$ penicillin, and $100 \mu \mathrm{g} / \mathrm{ml}$ streptomycin. Cells were maintained at $37^{\circ} \mathrm{C}$ in a humidified atmosphere of $5 \% \mathrm{CO} 2$.

\section{Flowcytometry}

Cells were stained with the PE-conjugated anti-Human PD-L1 cloneM1H1 (cBioscience, CA, USA). The cells' flow was assessed using Accuri ${ }^{\mathrm{TM}} \mathrm{C} 6$ (BD Biosciences, MI, USA). The data was analyzed by the Flowjo software (TreeStar, OR, USA) and the PD-L1 levels were determined by calculating the Median Fluorescence Intensity (MFI).

\section{Western blot analysis}

Western blot analysis was performed as previously described [27]. Briefly, whole cell lysates were collected by lysis buffer ( 25 mM HEPES $\mathrm{pH} 7.7$, $0.3 \mathrm{M} \mathrm{NaCl}, 1.5 \mathrm{mM} \mathrm{MgCl} 2,0.2 \mathrm{mM}$ EDTA, 0.1\% Triton X-100, $20 \mathrm{mM} \beta$-glycerophosphate, $1 \mathrm{mM}$ sodium orthovanadate, $1 \mathrm{mM}$ phenylmethylsulfonyl fluoride (PMSF), $1 \mathrm{mM}$ dithiothreitol (DTT), 10 $\mathrm{mg} / \mathrm{ml}$ aprotinin and $10 \mathrm{mg} / \mathrm{ml}$ leupeptin). Equal amounts of protein were resolved by electrophoresis on acrylamide gels (7.5 or $10 \%)$ and transferred to Immobilon-P nylon membrane (Millipore, Bedford, MA). The membranes were blocked with BlockAce (Dainippon Pharmaceutical, Co. Ltd., Osaka, Japan) for at least $2 \mathrm{~h}$, and probed with the indicated primary antibodies overnight, followed by the appropriate 
conjugated secondary antibodies. The bands were visualized using ECL reagents (Amersham Bioscience, Piscataway, NJ).

\section{Nuclear translocation analysis}

Nuclear translocation analysis was performed as previously described [28]. In brief, cells were suspended in $400 \mu \mathrm{l}$ buffer A (10 mM Hepes $\mathrm{pH} 7.9$, $10 \mathrm{mM} \mathrm{KCl}, 0.1 \mathrm{mM}$ EDTA, $0.1 \mathrm{mM}$ EGTA, $1 \mathrm{mM}$ DTT, $1 \mathrm{mM}$ PMSF, $20 \mathrm{mM} \beta$-glycerophosphate, $1 \mathrm{mM}$ sodium orthovanadate, $10 \mu \mathrm{g} / \mathrm{ml}$ aprotinin, and 10 $\mu \mathrm{g} / \mathrm{ml}$ leupeptin) with $25 \mu \mathrm{l} 10 \%$ Nonidet P-40 and the supernatants cytoplasmic fractions were collected. The nuclear pellets were suspended in $50 \mu$ l buffer B (20 mM HEPES pH 7.9, $0.4 \mathrm{M} \mathrm{NaCl}, 1 \mathrm{mM}$ EDTA, 1 mM EGTA, $1 \mathrm{mM}$ DTT, $1 \mathrm{mM}$ PMSF, $20 \mathrm{mM}$ $\beta$-glycerophosphate, $1 \mathrm{mM}$ sodium orthovanadate, 10 $\mu \mathrm{g} / \mathrm{ml}$ aprotinin and $10 \mu \mathrm{g} / \mathrm{ml}$ leupeptin) to yield the nuclear fractions. Immunoblot was conducted as indicated in the Western blot analysis section.

\section{Statistical analysis}

The results are expressed as MFI \pm S.E. Statistical significance was evaluated by either Student's t-test or one-way ANOVA followed by Bonferroni post-hoc test.

\section{Results}

\section{Aberrant EGFR activation drives higher expression of PD-L1 on NSCLC cell lines}

To first determine the association between the activation status of EGFR signaling pathway and PD-L1 expression on NSCLC, we analyzed the PD-L1 expression of NSCLC cell lines with different EGFR-mutation statuses. We compared the basal expression levels of PD-L1 between the EGFR mutated NSCLC cells (PC-9 cells) and the EGFR wild-type NSCLC cells (LU-99, A549, and PC-14 cells). As shown in Figure 1A, PD-L1 expression of PC-9 was significantly higher than those of EGFR wild-type cell lines (Figure 1A). In accordance with the higher PD-L1 expression, EGFR mutated PC-9 cells showed consistently higher expression of phosphorylated AKT, STAT3 and ERK along with EGFR compared to the other EGFR wild-type NSCLC cells though there were substantial differences in their total EGFR expression (Figure 1B). Given that PI3K/AKT, STAT3 and MAPK pathways are the downstream targets of EGFR signaling, these results indicate that the activation status of EGFR and its downstream pathways may be associated with the expression level of PD-L1 on NSCLC.
A
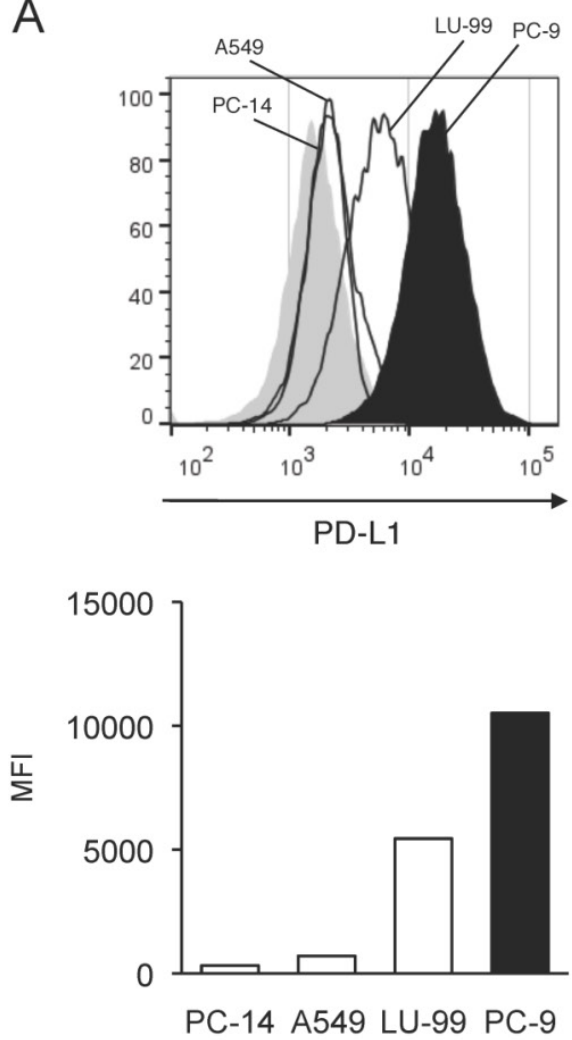

B
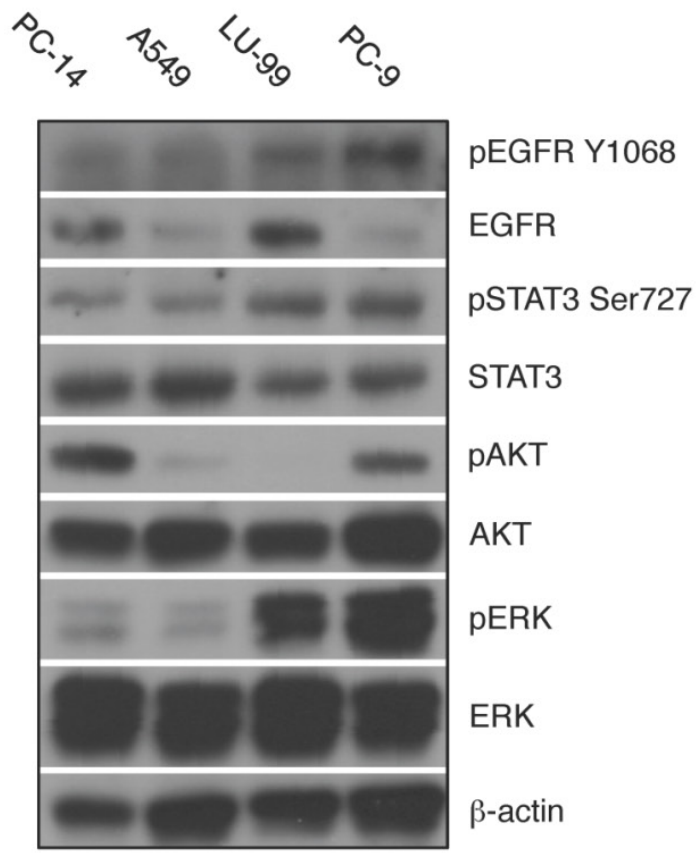

Figure 1. Aberrant EGFR activation status correlates with higher expression of PD-LI on NSCLC cell lines. (A) PD-L1 expression on EGFR wild-type (PC-14, A549, and LU-99, histograms with thin lines) or EGFR-mutant (PC-9, filled histogram) NSCLC cell lines are shown as histograms (upper panel) or as median fluorescence intensity (MFI) (lower panel) determined by flow cytometry. (B) The expression of indicated proteins in EGFR wild-type (PC-14, A549, and LU-99) or EGFR-mutant (PC-9) NSCLC cell lines are shown determined by western blotting. $\beta$-actin was used as an internal control. 


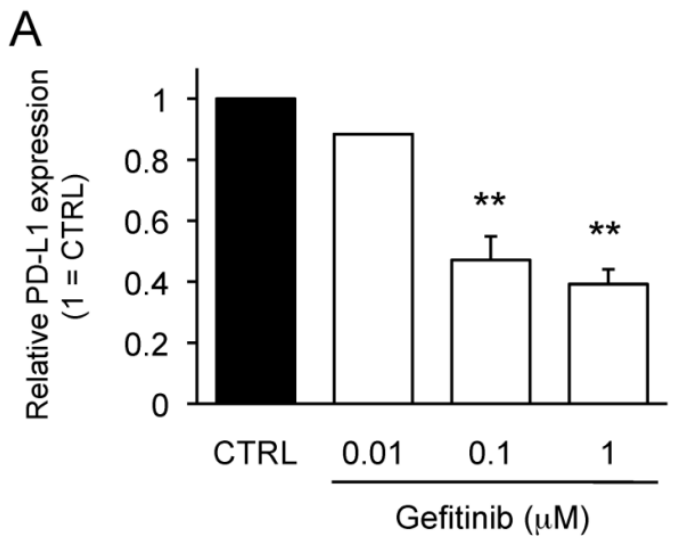

B

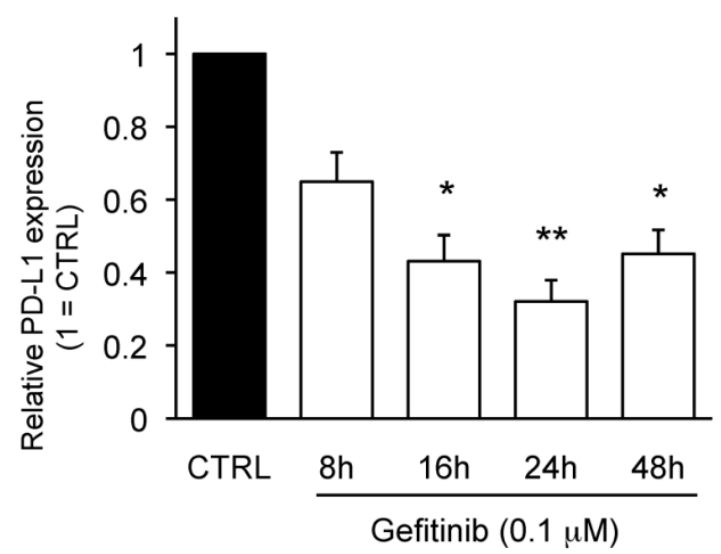

C

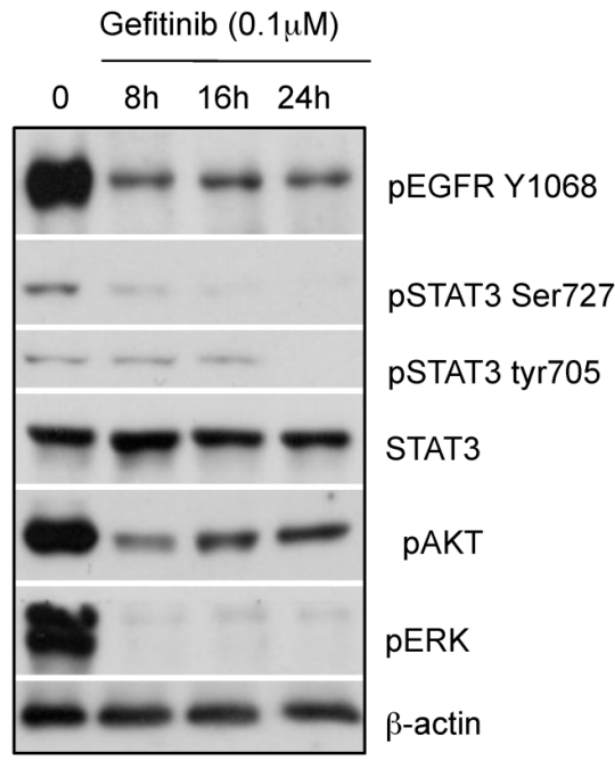

Figure 2. Effect of gefitinib on PD-LI expression of PC-9 cells and downstream pathways of EGFR signaling. (A) PC-9 cells treated with gefitinib at the indicated dose for 48 hours and the PD-L1 expression are shown as median fluorescence intensity (MFI) determined by flow cytometry. (B) PC-9 cells were treated with $0.1 \mu \mathrm{M}$ gefitinib for the indicated time and the PD-L1 expression are shown as median fluorescence intensity (MFI) determined by flow cytometry. (C) The expression of indicated proteins in PC-9 cells treated with $0.1 \mu \mathrm{M}$ gefitinib for the indicated time are shown determined by western blotting. $\beta$-actin was used as an internal control. Results from 3 independent experiments are shown and the significant differences between indicated groups are shown as $* p<0.05$ and $* * p<0.01$ compared to control group (CTRL). The control group was cells treated with the same amount of vehicle (DMSO).
We next examined the effect of gefitinib on PD-L1 expression of PC-9 cells bearing exon 19 mutation of EGFR, which determines the responsiveness to gefitinib. As shown in Figure 2A, the expression of PD-L1 on PC-9 cells was down-regulated by gefitinib treatment in a concentration-dependent manner. We also found that the time-dependent effect of gefitinib to down-regulate PD-L1 expression on PC-9 cells and the maximum response was observed at $24 \mathrm{~h}$ (Figure 2B). As shown in Figure 2C, we observed that PD-L1 downregulation upon gefitinib treatment was accompanied by the inhibition of pEGFR and its downstream pathway as seen in the pAKT, pERK and pSTAT3 expression. Collectively, these results strongly suggest that the aberrant EGFR activation can be an important driver for the higher expression of PD-L1 on NSCLCs.

\section{AKT-STAT3 pathway is responsible for regulating EGFR-driven PD-LI expression on NSCLC cells}

To determine a key EGFR-downstream pathway that is responsible for PD-L1 expression on NSCLCs, we used small molecule inhibitors for the PI3K/AKT and MAPK pathways. As shown in Figure 3A, the inhibitors for PI3K (LY-294002: LY), AKT (MK-2206: MK) and mTOR (Rapamycin: Rapa) significantly down-regulated the PD-L1 expression on PC-9 cells whereas the inhibitors for ERK (U0126: U) and JNK (SP600125: SP) did not. These results clearly indicate that PI3K/AKT pathway, but not MAPK pathway, can be responsible for PD-L1 expression on PC-9 cells. Furthermore, we observed the inhibitors for PI3K and AKT reduced the phosphorylation status of STAT3 to a similar extent as gefitinib treatment (Figure 3B). While the treatment with both AKT inhibitor (MK) and ERK inhibitor (U) showed reduced cytosolic STAT3 expression, AKT inhibitor, but not ERK inhibitor, inhibited the nuclear STAT3 expression in PC-9 cells (Figure 3C). Importantly, treatment with JAK/STAT3 inhibitor (JSI-124) significantly reduced the expression of PD-L1 on PC-9 cells (Figure 3D). Considering the combination of AKT inhibitor and STAT3 inhibitor did not show any additional effect on PD-L1 expression of PC-9 cells compared with either treatment alone (data not shown), the presented results indicate an importance of AKT-STAT3 pathway for regulating PD-L1 expression on EGFR-mutant PC-9 cells.

We then investigated whether AKT-STAT3 pathway is also responsible for PD-L1 expression in EGFR wild-type NSCLC cells upon the ligand activation of EGFR. As shown in Figure 4A, the expression of PD-L1 on EGFR wild-type LU-99 cells 
was significantly up-regulated by treating with recombinant EGF (rEGF) in a dose-dependent manner. In concert with the up-regulation of PD-L1 expression, we also detected the phosphorylation of AKT and STAT3 in LU-99 cells treated with rEGF A

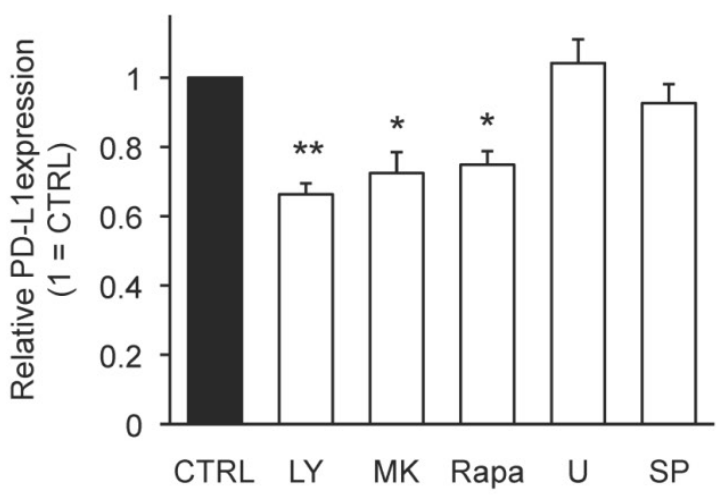

B

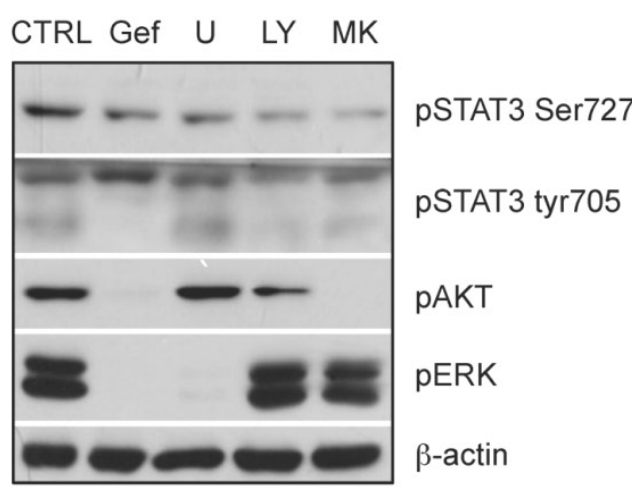

C

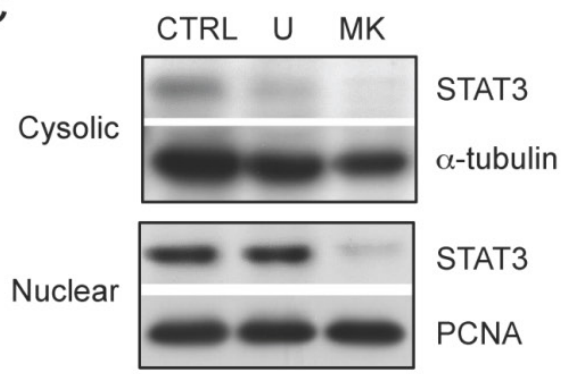

(Figure 4B). Importantly, the treatment with either AKT inhibitor or STAT3 inhibitor significantly inhibited the rEGF-driven PD-L1 up-regulation on LU-99 cells (Figure 4C) along with the inhibition of AKT or STAT3 phosphorylation, respectively (Figure 4D). Collectively, we clearly demonstrated that AKT-STAT3 pathway is responsible for regulating PD-L1 expression driven by both mutation-driven and the ligand-driven EGFR activation in NSCLC cells.

\section{Targeting AKT-STAT3 pathway bypasses gefitinib-resistance to regulate PD-LI expression on R-PC-9 cells}

NSCLC is known to frequently acquire resistance against gefitinib by developing an additional mutation, which eradicates its efficacy [24-26]. To investigate whether AKT-STAT3 pathway can be a potential target for modulating PD-L1 expression on NSCLCs acquiring gefitinib-resistance, we employed R-PC-9 cells, which is a gefitinib-resistant cell line of PC-9 by harboring T790M secondary EGFR mutation. There was no significant difference in the PD-L1 expression or the activation status of AKT and STAT3 between PC-9 and R-PC-9 (data not shown). Contrary to original PC-9 cells, R-PC-9 cells did not change their PD-L1 expression upon gefitinib treatment (Fig. 5A) confirming gefitinib-resistance of R-PC-9 cells even in PD-L1 regulation. Finally, we tested the effect of AKT inhibitor or STAT3 inhibitor on the expression of PD-L1 on R-PC-9 cells. As shown in Figure $5 \mathrm{~B}$, either a treatment with AKT inhibitor or STAT3 inhibitor significantly down-regulated the expression of PD-L1 on R-PC-9 cells. These results strongly indicate that the AKT-STAT3 pathway can be an attractive target to bypass gefitinib-resistance for regulating PD-L1 expression in NSCLCs.
Figure 3. AKT-STAT3 pathway is responsible for regulating mutant EGFR-driven PD-LI expression on NSCLC cells. (A) PD-L1 expression on PC-9 cells treated with the indicated inhibitors for 24 hours are shown as median fluorescence intensity (MFI) determined by flow cytometry. . doses of inhibitors use are as follow: LY (20 $\mu$ M LY-294002), MK (20 $\mu$ M MK-2206), RAPA (100 nM rapamycin), U (5 $\mu$ M U0126), and SP $(20 \mu \mathrm{M}$ SP600125) (B) The expression of indicated proteins in PC-9 cells treated with the indicated inhibitors for 24 hours are shown determined by western blotting. $\beta$-actin was used as an internal control. Pathways inhibition was confirmed by western blot for the indicated proteins (lower panel). (C) Cytoplasmic and nuclear fractions were collected and The expression of STAT3 protein either in cytosolic or nuclear fraction of PC-9 cells treated

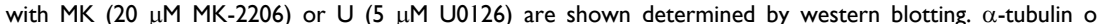
proliferating cell nuclear antigen (PCNA) was used as an internal control of cytosolic or nuclear protein respectively. (D) PD-L1 expression on PC-9 cells treated with JSI $(20 \mu \mathrm{M}$ JSI-124) for 24 hours are shown as median fluorescence intensity (MFI) determined by flow cytometry. Results from 3 independent experiments are shown and the significant differences between indicated groups are shown as $*^{*}<0.05$ and $* * p<0.01$ compared to control group (CTRL). The control group was cells treated with the same amount of vehicle (DMSO). 


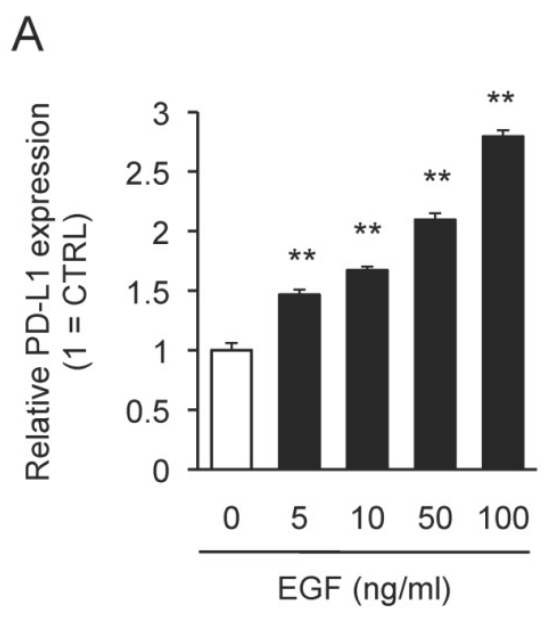

B
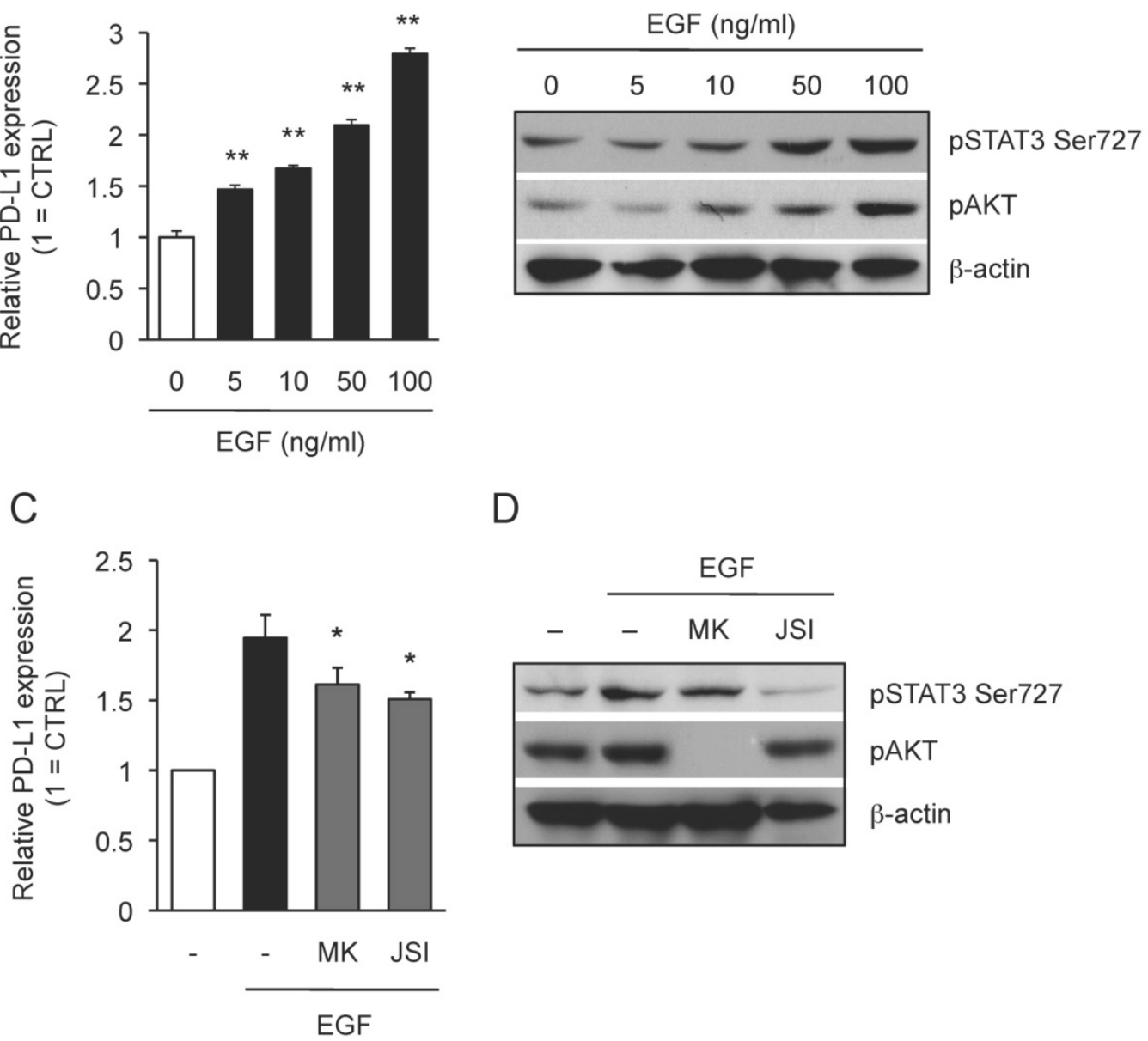

D

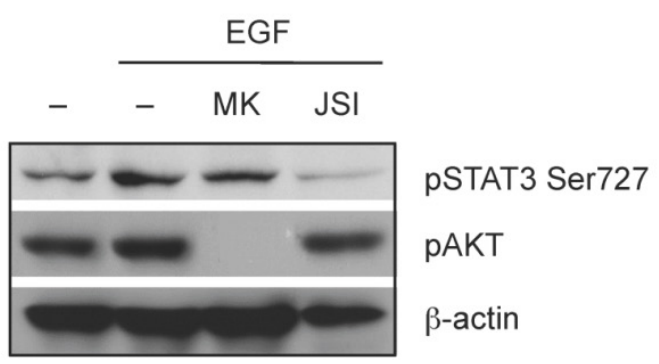

Figure 4. AKT-STAT3 pathway is responsible for regulating EGF-induced PD-L1 expression on NSCLC cells. (A) PD-L1 expression on LU-99 cells treated with human recombinant EGF at indicated dose for 24 hours are shown as median fluorescence intensity (MFI) determined by flow cytometry. Results from 3 independent experiments are shown and the significant differences between indicated groups are shown as $* * * p<0.01$ compared to non-treated group. (B) The expression of indicated proteins in LU-99 cells treated with human recombinant EGF at indicated dose for 24 hours are shown determined by western blotting. $\beta$-actin was used as an internal control. (C) PD-L1 expression on LU-99 cells co-treated with human recombinant EGF ( $50 \mathrm{ng} / \mathrm{ml})$ and MK (20 $\mu$ M MK-2206) or JSI (20 $\mu$ M JSI-124) for 24 hours are shown as median fluorescence intensity (MFI) determined by flow cytometry. (D) The expression of indicated proteins in LU-99 cells co-treated with human recombinant EGF (50 ng/ml) and MK (20 $\mu$ M MK-2206) or JSI (20 $\mu$ M JSI-124) for 24 hours are shown determined by western blotting. $\beta$-actin was used as an internal control. Results from 3 independent experiments are shown and the significant differences between indicated groups are shown as ${ }^{*} \mathrm{p}<0.05$ compared to group treated with EGF alone.

\section{Discussion}

In this study we identified that AKT-STAT3 pathway can be a novel downstream target of EGFR signaling for regulating PD-L1 expression on NSCLCs. Importantly, we further demonstrated the inhibition of either AKT or STAT3 activity can bypass a resistance to regulate PD-L1 expression in response to gefitinib treatment. Given the inhibition of both AKT and STAT3 did not show an additive effect on PD-L1 down-regulation, there should be the redundancy of AKT and STAT3 in the inhibition of PD-L1 expression on EGFR-activated cells. Considering recent attention of immunotherapy by modulating PD-1/PD-L1 interaction between the host immune system and cancer cells [5, 9], our present findings pave the way for sensitizing gefitinib-resistant NSCLCs to endogenous anti-tumor host immune response and/or $\mathrm{T}$ cell-dependent immunotherapy. Although it has been known that the inhibition of EGFR signaling can attenuate PD-L1 expression on NSCLCs [8, 20,29], there was no link between AKT-STAT3 pathways to regulate PD-L1 expression on NSCLCs as a downstream of EGFR signaling. Consistent with our findings, AKT/mTOR pathway is known to lead the activation of STAT3 [30] and PI3K-mediated STAT3 activation was shown to regulate PD-L1 in BRAF-mutant melanoma cells [31]. Additionally, AKT pathway was reported to contribute to PTEN-mediated PD-L1 regulation in breast cancer [18]. Furthermore, a direct binding of STAT3 to the PD-L1 promoter was reported in antigen-presenting cells [32] suggesting the direct regulation of PD-L1 expression by STAT3 at a transcriptional level.

In clinic, NSCLC patients initially respond to gefitinib or other EGFR TKIs, however the relapse of 
disease by acquiring drug-resistance through developing a second-site EGFR mutation in cancer cells has been a major obstacle [24-26]. Thus, it is important to develop an alternative approach to target such acquired drug-resistance of NSCLC. In this regard, we specifically showed that the inhibition of AKT-STAT3 pathway can down-regulate PD-L1 expression not only on PC-9 cells that respond to gefitinib, but also on the gefitinib-resistant R-PC-9 cells harboring an additional mutation. In addition to AKT-STAT3 pathway, p38MAPK pathway is known to be involved in EGFR signaling [33, 34]. Of note, p38 inhibitor did not show any effect on PD-L1 expression in R-PC-9 (data not shown) suggesting that p38MAPK pathway might not be involved in regulating PD-L1 expression as a downstream of EGFR signaling.

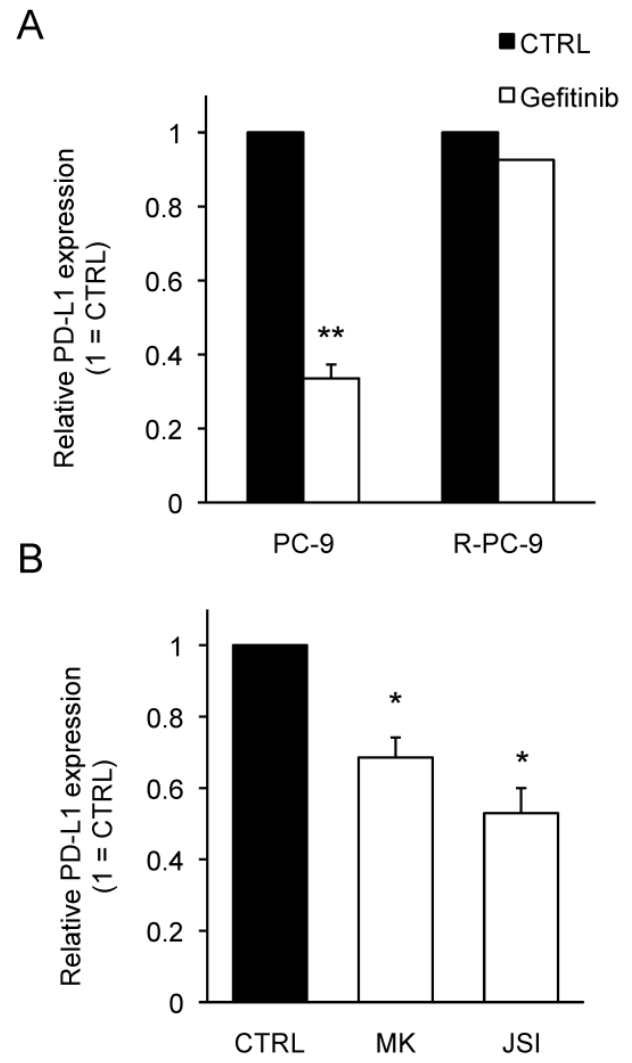

Figure 5. Inhibition of AKT-STAT3 pathway bypasses gefitinib-resistance to regulate PD-LI expression on R-PC-9 cells. (A) PD-L1 expression on PC-9 or R-PC-9 cells treated with or without gefitinib $(1 \mu \mathrm{M})$ for 48 hours are shown as median fluorescence intensity (MFI) determined by flow cytometry. (B) PD-L1 expression on R-PC-9 treated with MK $(20 \mu$ M MK-2206) or JSI $(20 \mu \mathrm{M}$ JSI-124) for 24 hours are shown as median fluorescence intensity (MFI) determined by flow cytometry. Results from 3 independent experiments are shown and the significant differences between indicated groups are shown as ${ }^{*} \mathrm{p}<0.05,{ }^{* *} \mathrm{p}<0.01$ compared to control group (CTRL). The control group was cells treated with the same amount of vehicle (DMSO).

While AKT-STAT3 pathway is widely known as important for cancer cell malignancy, accumulative evidences also suggest it can be a potential target for regulating host immune responses against cancer cells [35]. It has been reported that STAT3-deficiency in
CD8+ $\mathrm{T}$ cells leads to a proactive phenotype in response to its TCR stimulation [36] and STAT3 inhibits tumor accumulation of $\mathrm{CD}^{+} \mathrm{T}$ cells through regulating CXCR3/CXCL10 chemokine axis [37, 38]. Additionally, STAT3 inhibitor is known to potentiate the $\mathrm{T}$ cell cytotoxicity by attenuating regulatory $\mathrm{T}$ cell function [37, 38]. Furthermore, AKT pathway is shown to impair memory $\mathrm{CD}^{+} \mathrm{T}$ cell differentiation and its functional activity that can be cancelled by inhibiting AKT [39]. Collectively, targeting AKT-STAT3 pathway should be a promising strategy to potentiate anti-tumor immune responses by supporting both cancer cell intrinsic and cancer cell extrinsic mechanism as shown in PD-L1 down-regulation on cancer cells with an aberrant EGFR activity and to enhance host anti-tumor $\mathrm{T}$ cell immune responses, respectively.

\section{Acknowledgements}

We thank Hiroaki Sakurai for his discussion and suggestions, and Menolly Walter for her manuscript editing.

\section{Competing Interests}

The authors have declared that no competing interest exists.

\section{References}

1. Smyth MJ, Dunn GP, Schreiber RD. Cancer immunosurveillance and immunoediting: the roles of immunity in suppressing tumor development and shaping tumor immunogenicity. Adv Immunol. 2006; 90: 1-50.

2. Dunn GP, Old LJ, Schreiber RD. The immunobiology of cancer immunosurveillance and immunoediting. Immunity. 2004; 21: 137-48.

3. Marincola FM, Jaffee EM, Hicklin DJ, Ferrone S. Escape of human solid tumors from T-cell recognition: molecular mechanisms and functional significance. Adv Immunol. 2000; 74: 181-273.

4. Soria JC, Marabelle A, Brahmer JR, Gettinger S. Immune checkpoint modulation for non-small cell lung cancer. Clin Cancer Res. 2015; 21: 2256-62.

5. Zitvogel L, Kroemer G. Targeting PD-1/PD-L1 interactions for cancer immunotherapy. Oncoimmunology. 2012; 1: 1223-5.

6. Parsa AT, Waldron JS, Panner A, Crane CA, Parney IF, Barry JJ, et al. Loss of tumor suppressor PTEN function increases B7-H1 expression and immunoresistance in glioma. Nat Med. 2007; 13: 84-8.

7. Crane CA, Panner A, Murray JC, Wilson SP, Xu H, Chen L, et al. PI(3) kinase is associated with a mechanism of immunoresistance in breast and prostate cancer. Oncogene. 2009; 28: 306-12.

8. Akbay EA, Koyama S, Carretero J, Altabef A, Tchaicha JH, Christensen CL, et al. Activation of the PD-1 pathway contributes to immune escape in EGFR-driven lung tumors. Cancer Discov. 2013; 3: 1355-63.

9. Byrne KT, Vonderheide RH, Jaffee EM, Armstrong TD. Special Conference on Tumor Immunology and Immunotherapy: A New Chapter. Cancer Immunol Res. 2015.

10. National Cancer Institute. The Surveillance, Epidemiology and End Results (SEER) database.

11. Molina JR, Yang P, Cassivi SD, Schild SE, Adjei AA. Non-small cell lung cancer: epidemiology, risk factors, treatment, and survivorship. Mayo Clin Proc. 2008; 83: 584-94.

12. Konishi J, Yamazaki $K$, Azuma M, Kinoshita I, Dosaka-Akita $H$, Nishimura M. B7-H1 expression on non-small cell lung cancer cells and its relationship with tumor-infiltrating lymphocytes and their PD-1 expression. Clin Cancer Res. 2004; 10: 5094-100.

13. Mu CY, Huang JA, Chen Y, Chen C, Zhang XG. High expression of PD-L1 in lung cancer may contribute to poor prognosis and tumor cells immune escape through suppressing tumor infiltrating dendritic cells maturation. Med Oncol. 2011; 28: 682-8.

14. Robert C, Schachter J, Long GV, Arance A, Grob JJ, Mortier L, et al. Pembrolizumab versus Ipilimumab in Advanced Melanoma. N Engl J Med. 2015; 372: 2521-32 
15. Sullivan RJ, Flaherty KT. Pembrolizumab for Treatment of Patients with Advanced or Unresectable Melanoma. Clin Cancer Res. 2015; 21: 2892-7.

16. Sgambato A, Casaluce F, Sacco PC, Palazzolo G, Maione P, Rossi A, et al. Anti PD-1 and PDL-1 immunotherapy in the treatment of advanced non-small cell lung cancer (NSCLC): a review on toxicity profile and its management. Curr Drug Saf. 2015.

17. Bustamante Alvarez JG, Gonzalez-Cao M, Karachaliou N, Santarpia M, Viteri $\mathrm{S}$, Teixido C, et al. Advances in immunotherapy for treatment of lung cancer. Cancer Biol Med. 2015; 12: 209-22.

18. Mittendorf EA, Philips AV, Meric-Bernstam F, Qiao N, Wu Y, Harrington S, et al. PD-L1 expression in triple-negative breast cancer. Cancer Immunol Res. 2014; 2: 361-70.

19. Song M, Chen D, Lu B, Wang C, Zhang J, Huang L, et al. PTEN loss increases PD-L1 protein expression and affects the correlation between PD-L1 expression and clinical parameters in colorectal cancer. PLoS One. 2013; 8: e65821.

20. Chen N, Fang W, Zhan J, Hong S, Tang Y, Kang S, et al. Upregulation of PD-L1 by EGFR Activation Mediates the Immune Escape in EGFR-Driven NSCLC: Implication for Optional Immune Targeted Therapy for NSCLC Patients with EGFR Mutation. J Thorac Oncol. 2015; 10: 910-23.

21. Rusch V, Baselga J, Cordon-Cardo C, Orazem J, Zaman M, Hoda S, et al. Differential expression of the epidermal growth factor receptor and its ligands in primary non-small cell lung cancers and adjacent benign lung. Cancer Res. 1993; 53: 2379-85.

22. Akca H, Tani M, Hishida T, Matsumoto S, Yokota J. Activation of the AKT and STAT3 pathways and prolonged survival by a mutant EGFR in human lung cancer cells. Lung Cancer. 2006; 54: 25-33.

23. Sordella R, Bell DW, Haber DA, Settleman J. Gefitinib-sensitizing EGFR mutations in lung cancer activate anti-apoptotic pathways. Science. 2004; 305 : 1163-7.

24. Kobayashi S, Boggon TJ, Dayaram T, Janne PA, Kocher O, Meyerson M, et al. EGFR mutation and resistance of non-small-cell lung cancer to gefitinib. N Engl J Med. 2005; 352: 786-92.

25. Kaneto N, Yokoyama S, Hayakawa Y, Kato S, Sakurai H, Saiki I. RAC1 inhibition as a therapeutic target for gefitinib-resistant non-small-cell lung cancer. Cancer Sci. 2014; 105: 788-94.

26. Godin-Heymann N, Ulkus L, Brannigan BW, McDermott U, Lamb J, Maheswaran S, et al. The T790M "gatekeeper" mutation in EGFR mediates resistance to low concentrations of an irreversible EGFR inhibitor. Mol Cancer Ther. 2008; 7: 874-9.

27. Abdelhamed S, Yokoyama S, Refaat A, Ogura K, Yagita H, Awale S, et al. Piperine enhances the efficacy of TRAIL-based therapy for triple-negative breast cancer cells. Anticancer Res. 2014; 34: 1893-9.

28. Hafiyani L, Yokoyama S, Abdelhamed S, Hayakawa Y, Saiki I. Bufadienolides Overcome TRAIL Resistance in Breast Cancer Cells via JAK-STAT Pathway. Alternative \& Integrative Medicine. 2014; 3.

29. Lin K, Cheng J, Yang T, Li Y, Zhu B. EGFR-TKI down-regulates PD-L1 in EGFR mutant NSCLC through inhibiting NF-kappaB. Biochem Biophys Res Commun. 2015; 463: 95-101.

30. Yokogami K, Wakisaka S, Avruch J, Reeves SA. Serine phosphorylation and maximal activation of STAT3 during CNTF signaling is mediated by the rapamycin target mTOR. Curr Biol. 2000; 10: 47-50.

31. Jiang X, Zhou J, Giobbie-Hurder A, Wargo J, Hodi FS. The activation of MAPK in melanoma cells resistant to BRAF inhibition promotes PD-L1 expression that is reversible by MEK and PI3K inhibition. Clin Cancer Res. 2013; 19: 598-609.

32. Wolfle SJ, Strebovsky J, Bartz H, Sahr A, Arnold C, Kaiser C, et al. PD-L1 expression on tolerogenic APCs is controlled by STAT-3. Eur J Immunol. 2011; 41: 413-24.

33. Refaat A, Aminullah, Zhou Y, Kawanishi M, Tomaru R, Abdelhamed S, et al. Role of tyrosine kinase-independent phosphorylation of EGFR with activating mutation in cisplatin-treated lung cancer cells. Biochem Biophys Res Commun. 2015; 458: 856-61.

34. Nishimura M, Shin MS, Singhirunnusorn P, Suzuki S, Kawanishi M, Koizumi $\mathrm{K}$, et al. TAK1-mediated serine/threonine phosphorylation of epidermal growth factor receptor via p38/extracellular signal-regulated kinase: NF-\{kappa\}B-independent survival pathways in tumor necrosis factor alpha signaling. Mol Cell Biol. 2009; 29: 5529-39.

35. Kortylewski M, Kujawski M, Wang T, Wei S, Zhang S, Pilon-Thomas S, et al. Inhibiting Stat3 signaling in the hematopoietic system elicits multicomponent antitumor immunity. Nat Med. 2005; 11: 1314-21.

36. Yu CR, Dambuza IM, Lee YJ, Frank GM, Egwuagu CE. STAT3 regulates proliferation and survival of $\mathrm{CD} 8+\mathrm{T}$ cells: enhances effector responses to HSV-1 infection, and inhibits IL-10+ regulatory CD8+ T cells in autoimmune uveitis. Mediators Inflamm. 2013; 2013: 359674.

37. Kong LY, Wei J, Sharma AK, Barr J, Abou-Ghazal MK, Fokt I, et al. A novel phosphorylated STAT3 inhibitor enhances T cell cytotoxicity against melanoma through inhibition of regulatory $\mathrm{T}$ cells. Cancer Immunol Immunother. 2009; 58: 1023-32.

38. Yue C, Shen S, Deng J, Priceman SJ, Li W, Huang A, et al. STAT3 in CD8+ T cells inhibits their tumor accumulation by down-regulating CXCR3/CXCL10 axis. Cancer Immunol Res. 2015.

39. Kim EH, Sullivan JA, Plisch EH, Tejera MM, Jatzek A, Choi KY, et al. Signal integration by Akt regulates CD8 T cell effector and memory differentiation. J Immunol. 2012; 188: 4305-14. 